\title{
Recent developments in x-ray diagnostics for cryogenic and optically dense coaxial rocket sprays
}

\author{
Christopher D. Radke* \\ Propulsion and Power Division, NASA-Johnson Space Center, Houston, TX, 77058, USA \\ Alan L. Kastengren ${ }^{\dagger}$ \\ X-Ray Science Division, Argonne National Laboratory, Argonne, IL, 60439, USA \\ Terrence R. Meyer ${ }^{\ddagger}$ \\ School of Mechanical Engineering, Purdue University, West Lafayette, Indiana 47906, USA
}

The mixing and atomization of propellants is often characterized by optically dense flow fields and complex breakup dynamics. In the development of propulsion systems, the complexity of relevant physics and the range of spatio-temporal scales often makes computational simulation impractical for full scale injector elements; consequently, continued research into improved systems for experimental flow diagnostics is ongoing. One area of non-invasive flow diagnostics which has seen widespread growth is using synchrotron based x-ray diagostics. Over the past 3 years, a series of water and cryogenic based experiments were performed at the Advanced Photon Source, Argonne National Lab, on a NASA in-house designed swirl co-axial rocket injector, designed for operation using liquid oxygen and liquid methane in support of Project Morpheus. A range of techniques, such as x-ray fluorescence and time-averaged radiography were performed providing qualitative and quantitative mass and phase distributions, and were complemented by investigations using time-resolved radiography and white beam imaging, which provided information on breakup and mixing dynamics. Results of these investigations are presented, and conclusions regarding the viability of x-ray based diagnostics are discussed.

\section{Nomenclature}

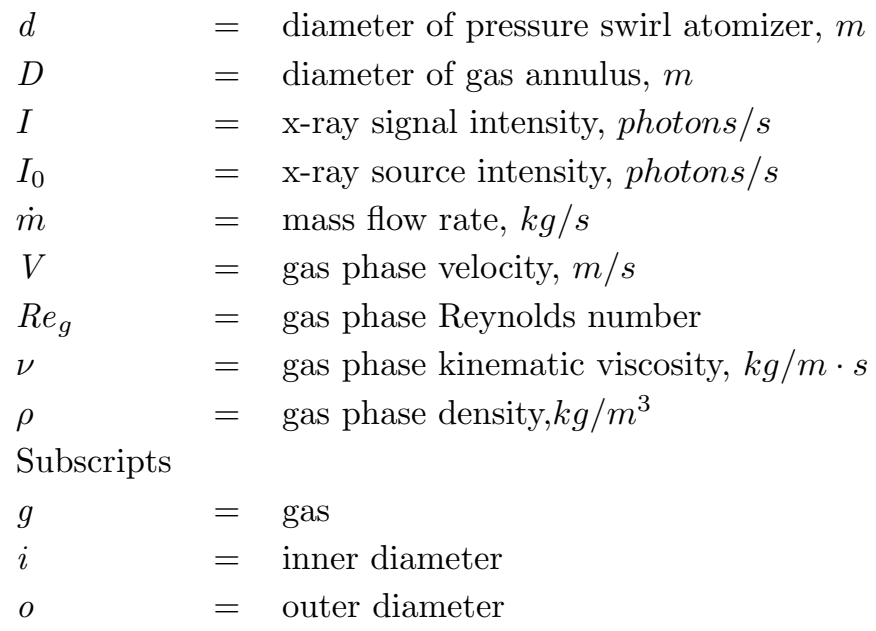

${ }^{*}$ Propulsion and Power Division, NASA-Johnson Space Center, Houston, TX, 77058, USA, Member AIAA, Christopher.D.Radke@NASA.gov

${ }_{\dagger}$ X-Ray Science Division, Argonne National Laboratory, Argonne, IL, 60439, USA

${ }^{\ddagger}$ School of Mechanical Engineering, Purdue University, West Lafayette, Indiana 47906, USA and Associate Fellow, AIAA 


\section{Introduction}

The atomization and breakup of a continuous fluid into a diffuse flow field of ligaments and droplets is of fundamental importance in many scientific and industrial processes. Applications such as spray cooling, aerosol drug delivery, and combustion processes, all rely on the rapid and uniform conversion via atomization of continuous liquids streams into diffuse highly atomized droplet fields. Given the prevalence of atomization and multiphase flow phenomena, continued investigations using both numerical and experimental methods. However, given the complexity of the relevant physics in many multiphase flows, and the wide range of relevant spatial and temporal scales required to be resolved for accurate simulation, accurate simulations using cutting edge numerical methods is often impractical.

As a consequence, new experimental methods continue to be developed to study these complex flow fields. Unfortunately, just as with numerical simulations, the complexity of these flow fields leads to difficulty probing these environments, even with modern diagnostic techniques. For 'optically dense' flow field, and flow fields with many phase boundaries, standard optical techniques such as shadowgraphy, Mie scattering and Doppler anemometry experience strong scattering at phase boundaries, resulting in limited data collection ability. ${ }^{1}$ To overcome these limitations, alternate diagnostics techniques such as SLIPI, Optical Connectivity, and Ballistic Imaging, have been pioneered for quantitative data collection in optically dense environments, ${ }^{2}$ but the viability of these techniques on the study of 'real' injectors (not modified to support the diagnostic) remains to be seen. Additionally, the ability of these techniques to quantify certain quantities of interest, such as ability absolute mass distributions, remains to be seen.

An alternative approach to optical based diagnostics for the study of fluid flows has been built around the utilization of x-rays. Unlike visible light wavelengths, which are susceptible to strong scattering (particularly at phase interfaces), ${ }^{3} \mathrm{x}$-rays predominantly interact with fluid media via photoelectric absorption and fluorescence. Consequently, line-of-sight x-ray diagnostics allow for interrogation of optically dense flow fields. In this vein, a range of diagnostics have been developed using both tube source x-rays and synchrotron based x-rays. ${ }^{4-7}$

Within the field of x-ray diagnostics, several separate techniques have to date been utilized. Among the most common techniques, radiography, is relies on the absorption of incident radiation in accordance with Beers' law, often given as:

$$
\frac{I(\lambda, z)}{I_{0}(\lambda, z)}=e^{-\int \mu(z) d z}
$$

Here $\mu$ is the attenuation coefficient defined as a product of the attenuation cross-section and number density, $z$ is the coordinate along the beam path, and $y$ and $z$ are the transverse coordinates.

In short, Beers' law states that if the absorption coefficient and the X-ray intensity both before and after the sample are known, the path length through the sample as a function of time be computed. It should be noted however, that the attenuation coefficient of most materials and fluids is a strong function of wavelength, and use of polychromatic x-ray sources results in difficult conversion to quantitative path length, often requiring modeling. However, for monochromatic beams, the wavelength dependence becomes irrelevant, and quantitative results are straight forward. Rearranging the previous result, we can produce the useful equation:

$$
l(t)=\frac{1}{\epsilon(\lambda) \rho} \ln \left(\frac{I_{0}(\lambda, z=0)}{I(\lambda, z=l)}\right)
$$

Where $\rho$ is the line of sight averaged density. For a highly focused monochromatic beam of x-rays, the line of sight technique can accurately measured probe volumes on the order of several microns and time rates of $\mathrm{MHz}$ can be recorded.

In addition to x-ray radiography, x-ray fluorescence, has recently been demonstrated and used to allow independent phase tracking in many multiphase flow experiments. ${ }^{8-11}$ In x-ray fluorescence, illumination by x-rays with sufficient energy causes photoionization of an electron in the shell, leading to the emission of $\mathrm{x}$-rays at specific energies associated with an electron filling the vacancy from a nearby shell. X-ray fluorescence can be used to identify specific elements because the fluorescence photon energy is dependent on the atomic number of each element. As such, x-ray fluorescence is sensitive to the density of the targeted element but is insensitive to chemical bonding, pressure, temperature, or physical phase. As the emission is in the x-ray regime, it is not attenuated significantly by scattering from phase interfaces and has shown to be useful diagnostic 
Finally, x-ray imaging has also seen recent development and advancements in both tube sources and at the APS - 7BM. ${ }^{12-17}$ Many previous efforts utilizing x-ray imaging utilized phase contrast imaging and tube sources, though recent facility upgrades have has made imaging at the APS - 7BM possible.

\section{Experimental Setup}

\section{A. Diagnostic Setup}

All of the experiments performed within this work were performed at the Advanced Photon Source (APS) at Argonne National Lab. The x-ray source at the APS is a bending-magnet, which can provide both a narrowband monochromatic x-ray beam for line of sight investigations of radiography and fluorescence. 2.

The beamline at the APS consists of two 'hutches' or enclosures each with different functions. The first enclosure, named 7-BM-A, takes the broad band x-ray beam from the source and conditions the beam for use in the second enclosure, named 7-BM-B. The conditioning of the beam is dependent on the application, particularly depending on experiments requiring either monochromatic focused x-rays or polychromatic 'white beam'. A diagram of the hutch is shown in 2.

For experiments requiring narrowband, focused x-rays, the broadband beam is allowed to enter the facility through a series of slits that provide some level of filtering of the source beam. The beam then enters a monochromater $(\Delta \mathrm{E} / \mathrm{E}=1.4 \%)$ to create a nearly collimated monochromatic beam. The beam is then focused using a pair of $300 \mathrm{~mm}$ long mirrors in a Kirkpatrick-Baez geometry at 5 mrad grazing angle. Using this setup, the APS is capable of producing beam energies between $\approx 6-15 \mathrm{keV}$. The flux of the beam is approximately $1.6 \mathrm{e} 11$ photons/s at $15 \mathrm{keV}$. Once focused, the resolution of the monochromatic beam, normal to the beam direction, is $5 \mu \mathrm{m} \times 7 \mu \mathrm{m}$ full width at half maximum focus of the beam. The divergence of the beam is approximately $2 \mathrm{mrad}$ (vertical) $\mathrm{x} 3 \mathrm{mrad}$ (horizontal).

For narrowband radiography measurements, incident focused x-rays first pass through focusing mirrors, before passing through a diamond crystal diode to measure incident radiation intensity. After leaving the diamond diode, the x-ray beam transverses through the spray to a silicon PIN diode detector. The PIN diode is an unbiased $300-\mu \mathrm{m}$ thick silicon detector, which can absorb $\approx 89 \%$ of incident transmitted radiation.

For many narrowband x-ray experiments, tracer fluorescence signals were simultaneously recorded with radiography measurements. For all fluorescence measurements, a silicon drift diode (SDD) integrated the fluorescence signal along the $\sim 1 \mathrm{~cm}$ long probe volume at a distance of approximately $15 \mathrm{~cm}$. To reduce slight elastic scattering from the excitation beam, which is comparable to the fluorescence signal but spectrally distinct, the SDD was positioned at approximately 90 degrees and placed behind a Teflon collimator.

For time-averaged measurements, the PIN diode output was amplified with a trans-impedance amplifier and normalized by the beam intensity monitor. Absorption measurements were collected at $1 \mathrm{MHz}$ for 2 second exposures on a digital oscilloscope sampling at $6.25 \mathrm{MHz}$. For time-resolved measurements, the PIN diode output was amplified with a transimpedance amplifier, recorded on a digital oscilloscope, and normalized by a diamond crystal beam monitor location prior to the spray to correct for modulations in beam intensity. Radiography data was recorded with a PIN diode using a multipole filter at $1 \mathrm{MHz}$ and recorded in 2 second exposures on a digital oscilloscope sampling at $1.25 \mathrm{MHz}$.

For polychromatic x-ray imaging at the APS, several changes in experimental setup were made. Most notably, the monochromator and focusing mirrors were removed, allowing the source beam to diverge from its source point to the experiment. The source point has a size of $80 \mu \mathrm{m} \mathrm{x} 200 \mu \mathrm{m}$ full width at half maximum, and the distance between the source point and the experiment were approximately $36 \mathrm{~m}$ from the experiment. As a result of the lack of conditioning optics, the resulting beam used for the studies lacks many optical aberrations, has significantly wider field of view, and has a greatly increased flux. In fact, the raw power of the beam, approximately $0.6 \mathrm{~W} / \mathrm{mm}^{2}$, can readily damage equipment and is stopped with a set of cooled lead blocks at the end of the hutch. Additionally, to prevent degradation of equipment due to the high-flux beam, a rotating chopper wheel was placed in the line of the beam to reduce the exposure of the beam prior to data collection. Additionally, lead shielding was placed around the imaging equipment to shield from scattered x-rays. Using this setup, the APS is capable of producing beam energies between approximately $1-200 \mathrm{keV}$. 


\section{B. Injector, instrumentation, and operation background}

The injector studied was a gas-liquid swirl coaxial injector designed to produce $110 \mathrm{~N}$ thrust utilizing liquid oxygen and gaseous methane propellants. The injector used was designed as a modular swirl coaxial rocket engine and as a potential successor to the HD series of rocket engines used in the Project Morpheus main engine. In the injector, a centrally located pressure swirl atomizer discharges a hollow cone into a circular annulus of flowing gas. The pressure swirler has an inner diameter of $3.2 \mathrm{~mm}$ and an outer diameter of 6.8 $\mathrm{mm}$. The distance between the pressure swirl atomizer and the injector exit is $10.2 \mathrm{~mm}$. The outer diameter of the gas annulus is interchangeable, with an outer diameter of $10.5 \mathrm{~mm}, 12 \mathrm{~mm}$ and $13.5 \mathrm{~mm}$. A diagram of the injector is shown in Fig. 1.

For all experiments, the injector was mounted to a vibrationally isolated, two dimensional translation stage, which translated the injector in a two-dimensional plane around the beam. To facilitate an investigation of both the center liquid phase and the combined gas-liquid atomizing flow field, the injector studied was designed to operate using only the central liquid pressure swirl atomizer (with it's geometry exposed), as well as in a combined gas-liquid flow field.

During the experiments, a range of instrumentation was used to collect fluid state properties. All instrumentation was recorded by a digital oscilloscope sampling at $1 \mathrm{~Hz}$ to time synchronize instrumentation data with collected x-ray diagnostics information. For all experiments, volumetric turbine flow meters, pressure transducers, and thermocouples were used to measure fluid state properties. Omega PX319 series pressure transducers are shown to have a quoted uncertainty of $\pm 0.25 \%$ Full Scale $( \pm 8.6 \mathrm{kPa})$ accounting for linearity, hysteresis and repeatability. The Omega type $\mathrm{K}$ thermocouples are shown to have a manufactured quoted uncertainty of $0.75 \%$. A Turbines Inc. liquid flow meter was used to record liquid flow rates for cryogenic fluids. The flow meter has a manufacture quoted uncertainty of $\pm 0.1 \%$ repeatability within range and $\pm 0.5 \%$ linearity within approved range. To measure gas flow, a Hoffer HO1/2X1/4A turbine gas flow meter was used to record all gas flow rates for all gases used within the study. The gas flow meter had a manufacturing quoted uncertainty of $\pm 0.1 \%$ repeatability within range and $\pm 1.0 \%$ linearity within range. To access different locations in the spray, the injector was translated horizontally and vertically on vibration isolated translation stages.

For water experiments, two methods for injecting the water were provided. The primary method used was pressurizing a bottom-feeding ASME pressure vessel that was connected to the injector via flexible nylon tubing. Once the flow was atomized downstream of the injector it was collected and recycled in stainless collection tank. While this method had the advantage of allowing for very steady flow, free to pressure transients, it only provides limited run time. The second method used in the experiments was to make a continuous "closed loop" system utilizing an electrically powered twin gear pump pushing through a liquid regulator. This method allowed for continuous running, and no pressure transients were observed in any flow data, though fluorescence tracer concentration was observed to vary slightly at collection of the spray was not $100 \%$ complete.

To more accurately simulate the relevant fluid mechanics of the injector as it would operate during combustion and to provide a more relevant environment for the demonstration of diagnostics, a study was performed using cryogenic fluids. Within the experiments, studies were performed using liquid nitrogen and liquid argon as simulants for liquid oxygen. The choice of these fluids was based on their density, fluorescence properties, and availability.

In order to accurately and safely simulate a cryogenic flow field, subcritical cryogenic liquids were injected into the injector via pressurized dewars. The dewars were pressurized with gaseous helium and heavily instrumented with temperature and pressure transducers to ensure fully subcooled flow entering the injector. As with experiments with water, the system was fully instrumented with volumetric flow meters on both liquid and gas inlet systems. To thermally condition, or "chill-in" the system prior to data collection, a set of two dewars were plumbed together in parallel that could be operated independently. Using this set up, the first dewar could be used to thermally condition prior to data collection, and the second could be used to provide the liquids needed for the experiments. For all cryogenic experiments, fully steady state conditions were obtained prior to data collection.

For all experiments, high speed visible light shadowgraphs were collected in addition to x-ray data from radiography, and fluorescence if relevent. A picture of the experimental setup for cryogenic operation is shown in Fig. 3. Taking advantage of the modularity of the injector, scans of just the liquid only pressure swirl atomizer flow field were able to be performed in addition to the combined liquid and gas atomized flow field by removing the gas co-flowing annulus. 


\section{Results and Discussion}

A first series of experiments were performed to characterize mass distributions using narrow-band timeaveraged radiography. To accomplish this, time-averaged scans were performed for both water and cryogenic spray experiments. Example visible light shadowgraphs of the liquid only pressure swirl atomizer flow in cryogenic operation is shown in Fig. 4, and of the combined liquid gas flow field for water and cryogenic experiments in Figs. 5 and Fig. 6 respectively.

To process the data and convert it into quantitative path length, different values for attenuation coefficient were used depending on beam energy and flow media. Example values are given below in Table 1.

Table 1. Flow constituents and attenuation coefficient

\begin{tabular}{l|l|l|}
\hline Flow Constituents & Beam Energy $(\mathrm{keV})$ & Attenuation Coefficient $\left(\mathrm{cm}^{2} / \mathrm{g}\right)$ \\
\hline Water & 7.1 & 14.85 \\
\hline$N_{2}$ & 6.6 & 13.54 \\
\hline$N_{2} N_{2} \% \mathrm{Kr}$ & 15.0 & 1.236 \\
\hline $99 \%$ & 15.0 & 2.392 \\
\hline $\mathrm{Ar}$ & 6.6 & 200.3 \\
\hline $\mathrm{Ar}$ & 15.0 & 19.83 \\
\hline $\mathrm{Air}$ & 6.6 & 17.07 \\
\hline Air & 7.1 & 13.72 \\
\hline Air & 15.0 & 1.56 \\
\hline
\end{tabular}

A first series of experiments was performed on the pressure swirl atomizer. For water scans, a closed loop cycle was designed facilitating continuous operation and scanning. This allowed for highly spatially resolved data collection at many data points. The flow field, which is a hollow cone, has a pronounced edge effect as the beam propagates through the edge of the spray. An example plot demonstrating the level of spatial resolution capable is shown in Fig. 7. As can be observed, high resolution line scans were taken at axial distances from $0.1 \mathrm{~mm}$ to $10 \mathrm{~mm}$ from the injector exit. As can be observed, the distribution of mass is shown to have very strong peaks as the beam is shown to propagate through the edge of the flow. In the centerline areas of the flow, the liquid path length can be shown to decrease as increasing distance from the injector exit as expected with a uniform mass distribution.

Following these first sets of experiments using time-averaged radiography for water simulant flows, a second round of experiments was performed with the injector being subjected to cryogenic flow conditions using liquid argon and liquid nitrogen.

As was implored in water flow experiments, Beer's law was utilized to obtain quantitative measures of path from the radiography data. Observation of Beer's law reveals that the conversation from absorption to path length is a strong function of density, which is particularly relevant in the study of cryogens. For single phase flows, such as our water experiments, the density terms is constant throughout the flow field. However, for the study of cryogens, the density of the flow can not be assumed to be constant throughout the flow region. As a consequence of this, quantitative calculations of path are only valid so long as the state properties can be accurately estimated.

In order to get an idea of potential evaporation effects, vertical scans were performed using liquid argon and nitrogen to estimate how the path length is observed to change. Vertical line scans were found to be particularly helpful in understanding the evolutionary dynamics of the fluid flow. An example of this type of scan is shown in Fig. 8 for centerline liquid argon flows from the pressure swirl atomizer.

In the processing of this data however, it was also important to accurately calibrate the line scans since the entirety of the scan was within the flow, and no 'air' scans were taken. For data processing shown, the vertical line scans were calibrated using horizontal scan data taken for nearly identical flow conditions (and taken in nearly successive scans).

To characterize this phenomena further, the mean and standard deviations of signals were analyzed for nitrogen and argon scans. Example horizontal line plots taken across regions of the pressure swirler flow field are shown in Figs. 9 and 10. Note the arbitrary units of extinction lengths as these calculations are not absolute units. As can be observed, flows of liquid nitrogen are shown to contain much less variation than 
comparable liquid argon flows in large part due to the significant amount of absorption of the beam. Since liquid argon has both a larger attenuation coefficient and higher density, the transmission of the beam was monitored to ensure adequate signal was received at the PIN diode. However, for several scans, the flow field was observed to nearly attenuate the entire beam and resulted in increased signal variation for dense regions of the spray.

Once the capability of the diagnostic was demonstrated for a single phase and geometrically simple flow, its utility in the study of a multiphase rapidly atomizing flow was investigated. As with before, a first series of experiments was performed using water and air or gaseous nitrogen as the atomizing gas. This experimental setup has the advantage of operating in a 'closed loop' configuration, allowing for scans with longer signal integration times and mapping at high spatial resolutions. Non-dimensional parameters, such as the Reynolds number, are extremely helpful in defining flow dynamics, and is defined for the purposes of the experiment as:

$$
R e_{\text {gas }}=R e_{g}=\frac{\rho V_{\text {gas }}\left(D_{\text {outer }}-D_{\text {inner }}\right)}{\nu}
$$

Where $\rho$ is the calculated gas phase density, $V_{\text {gas }}$ is the gas velocity, $D_{\text {outer }}$ is the outer annulus diameter, $D_{\text {inner }}$ is the inner annulus of the injector, and $\nu$ is the calculated viscosity of the gas.

A first example plot of a combined gas-liquid coaxial flow is shown in Fig. 11. Observed, high resolution line scans of the flow field show a narrow and significant edge structure on both sides of the spray, with a smooth and continuous central region. For these scans, the horizontal spacing of the scan locations was 0.1 $\mathrm{mm}$, and 145 total line scans were taken across the spray. Additionally, two nearly symmetrical 'bumps' near the centerline of the spray. Since the technique is line of sight and path averaged, the approximate liquid film thickness is most accurately defined at the spray centerline (position $0.0 \mathrm{~mm}$ ), at a local minimum of the spray, where the liquid film thickness is defined one half of the absorption measured liquid path length through the center of the spray without co-flowing gas.

Once the utility of the diagnostic was proven on water flows, it was again applied towards the utilization of cryogenic flows. Unlike the water based experiments, the cryogenic experiments required the operation of a cryogenic dewar and had limited run time. As a consequence, the number of data points taken at each flow condition was particularly limited. Further, the flow rate of the cryogenic fluid was limited to a range that produced fully subcooled cryogenic fluid at the injector, but did not require an excessive amount of helium pressurant within the dewar. It is also worth noting that since the flow field is comprised of only nitrogen at a constant incident wavelength (having constant attenuation coefficient), the calculation of the liquid path length becomes a function of the calculation of density between the phases. Since the density of liquid nitrogen is approximately 700 times larger than that of gas nitrogen, the path length due to gas phase nitrogen becomes trivial and the quantitative measure of path length can be inferred to be directly a contribution from the liquid phase.

Shown in Fig. 12, an example plot of quantitative path length is presented for a liquid nitrogen mass flow rate of $87.7 \mathrm{~g} / \mathrm{s}$ and a co-flowing gas of nitrogen at a Reynolds number of $R e_{g} \approx 11,000$ at $\mathrm{X}=0.5 \mathrm{~mm}$ $\left(x / D_{0}=0.05\right)$ from the injector exit. As can be observed, the mass distribution is shown to resemble that of the water spray flow field with edge peaks.

Following investigations of liquid path length, a follow up investigation was performed aimed at understanding quantitative phase distributions of both liquid and gas phases using tracer fluorescence in both liquid and gas phases. Full details of these experiments are reported in ${ }^{9}$ and only a brief summary is detailed here. For these experiments, the incident X-ray beam was set to $10.1 \mathrm{keV}$ enabling significant attenuation in the liquid phase while also exciting both liquid and gas-phase X-ray fluorescence. To excite liquid fluorescence, $200 \mathrm{ppm}$ of nickel sulfate hexahydrate $(7.5 \mathrm{keV} \mathrm{K \alpha})$ and $200 \mathrm{ppm}$ of zinc sulfate heptahydrate $(8.6 \mathrm{keV}$ $\mathrm{K} \alpha$ ) were dissolved in water and gas phase fluorescence of argon $(2.9 \mathrm{keV} \mathrm{K} \alpha)$ was collected simultaneously.

To obtain a quantitative path length of the separate phases, a multi-step procedure was performed. First, a cuvette study was performed and a correlation equation between the measured fluorescence yield and x-ray absorption for different known path lengths of the dual-liquid tracer solution was calculated. Next, the injector was operated at different conditions and line scans were performed collecting absorption and fluorescence yields at different locations across the scan. Following this, the quantitative path length of the liquid phase was able to be calculated from the measured liquid fluorescence tracer yield. By then subtracting the calculated liquid path length (from the liquid fluorescence) from the measured total attenuation, the differential amount can be inferred to be a quantitative measure of the gas phase. 
For cryogenic experiments, information regarding the phase distribution of the gas and liquid phases was also desired, though the addition of tracer fluorescence to the cryogenic fluids was deemed impractical. As a consequence, qualitative information regarding the liquid phase was measured through measurement of gas-phase only tracer fluorescence. An example figure is shown in 13 for liquid water and a $99 \%$ blend of $\mathrm{N} 2$ and a $1 \%$ blend of $\mathrm{Kr}(12.6 \mathrm{keV} \mathrm{K} \alpha)$. For moderate beam energies $(\approx 15 \mathrm{keV})$, krypton has a low attenuation coefficient and does not significantly attenuates the incident probe beam. Additionally, its high-energy fluorescence emission also experiences limited self-reabsorption as it propagates through the spray. The major draw back is its inability to provide qualitative measures of gas phase path length without separate calibration scans to correlate measured counts to path length. Since the fluorescence production is concentration dependent, the results do provide insight into relative concentration at areas within the spray, but with arbitrary units. Follow on investigations using krypton fluorescence with cryogenic fluids were also performed and are fully detailed in Radke et al. ${ }^{18}$

Once investigations into liquid path length, as well as mass and phase distributions were performed, a follow up investigation into the utility of time-resolved radiography to facilitate measurements of mixing was performed. Due to the high optical densities involved in many cryogenic spray, little is known about the evolution of mixing dynamics of these types of sprays. In an attempt to better quantify this mixing, timeresolved traces of x-ray attenuation were processed using a custom designed signal processing algorithm using Matlab. Results of these investigations demonstrated viability of time-resolved radiography in the quantifying turbulent mixing within the spray as fully detailed in. ${ }^{18}$ Notably, it was observed that for highly turbulent conditions, spectral evolution of time resolved trances were found to transition to a pronounced $-5 / 3$ slope in accordance with Kolmogorov's classical theory of homogeneous and isentropic turbulence.

Further investigation of the technique was used to probe the evolution of the flow dynamics by comparing the spectra at different spatial locations, but at identical flow conditions, as shown in Fig. 14. The measured spectra were largely the same, however, distinct levels of beam attenuation was observed particularly for frequencies less than 1e4. For $x / D_{O}=0.048$, the attenuation at $4883 \mathrm{~Hz}$ was measured to be nearly half of that of $x / D_{O}=1.00$ signal, 21.53 compared to 41.48 . This indicates less pronounced large scale motion, as would be expected for near nozzle exit flow, since the gas phase has had limited time to mix and expand throughout the flow. However, at higher frequencies, the levels of attenuation for the different conditions converge, indicating small scale motion is consistent, as would be expected.

An additional summary plot, Fig. 15, facilitates a more complete comparison between the diagnostic application at different beam energies and media. Argon attenuation at $6.6 \mathrm{keV}$, can be observed to track the turbulent spectra over the frequency range of interest, up until the Nyquist frequency. For liquid phase attenuation measurements, nitrogen attenuation was observed to capture spatio-temporal dynamics, though the signal begins to lose resolution at frequencies greater than 2.25e5. Finally, attenuation from the liquid phase at $15.0 \mathrm{keV}$ is extremely noisy with limited value compared to the $6.0 \mathrm{keV}$ signal. Calculations of relative beam attenuation at $\approx 8500 \mathrm{~Hz}$ show the argon attenuation at $6.6 \mathrm{keV}$ to be a factor of 49 larger than the nitrogen at $6.6 \mathrm{keV}$ and 648 times larger than nitrogen at $15.0 \mathrm{keV}$.

Following investigations which utilized monochromatic time-averaged and time-resolved scans, a follow on series of experiments were performed aimed at investigation of two dimensional transient fluid phenomena, such as ligament breakup and droplet formation using x-ray radiation. Recent upgrades at the Sector 7 bending-magnet beamline at the Advanced Photon source have recently allowed for a high intensity continuous wave source of broadband $\mathrm{x}$-rays with comparatively large field of view.

In the research campaign, a recently developed two-dimensional x-ray radiography technique was applied to the study of transient fluid structure during spray atomization at speeds of $120 \mathrm{kHz}$, as fully detailed in Halls et al. ${ }^{17}$ Radiographic images collected also revealed complex fluid structure within the primary atomization region of the spray, such as hollow droplets and stable mixed phase regions. An example plot is shown in Fig. 16. Experiments utilizing the technique were performed for a range of flow conditions providing insight into the breakup processes at different atomization regimes.

Additionally, efforts to quantitative model the transmission of the polychromatic beam through various optical aspects of the spray were performed using XOP, ${ }^{19}$ allowing an effective polychromatic x-ray attenuation coefficient through the system to be calculated. Using this attenuation coefficient, quantitative estimates of liquid path length were able to be performed on observed flow features, such as film thickness, ligament sizes, and hollow droplets. 


\section{Conclusion}

A recent research campaign at the 7-BM beamline at the Advanced Photon Source, Argonne National Lab was performed using a NASA designed swirl-coaxial injector designed in support of NASA's Project Morpheus. Investigations were performed using both water based experiments, as well as subcritical cryogenic fluids, such as liquid nitrogen and liquid argon, in addition to co-flowing gases of gaseous nitrogen, gaseous argon, and gaseous nitrogen with tracer fluorescence added. Results of the investigations demonstrated the viability of both narrow band and polychromatic x-ray diagnostics in the study of optically dense and dynamic flow fields.

Using monochromatic x-ray diagnostics, quantitative measurements of path length were measured to water and cryogenic flow fields at various locations within the spray for both pressure swirl atomizer flow fields, as well as combined liquid-gas flow fields. Further, argon and krypton fluorescence scans were performed allowing for both qualitative and quantitative measurements of phase distributions within the flow field. Additionally, time-resolved radiography scans were performed with results demonstrating the utility of time-resolved traces in facilitating measurements of mixing. During the investigations, the observation of spectral slope evolution to a $-5 / 3$ value at highly turbulent conditions, in agreement with Kolmogorov's classical theory of homogeneous and isentropic turbulence, provided further insight into the potential value of x-rays for the study of turbulent fluid flows.

Finally, a series of investigations using polychromatic x-rays were performed to investigate breakup dynamics and mechanisms in the near injector region of the spray. Observations of complex flow features, such as hollow droplets and liquid films with stable mixed phase regions, were routinely found. Models used to calculate polychromatic attenuation coefficients were also created, facilitating quantitative measurements of path length for observed liquid structures.

Combined, the recently demonstrated set of x-ray based diagnostics highlight the viability and utility of x-ray based diagnostics for the study of optically dense, multiphase and cryogenic based injector flows. Considering the very limited quantitative data sets available for cryogenic injector flows, particularly on full scale injector elements, the techniques showcased provides a new pathway for experimental model validation of this family of injector flows.

\section{Acknowledgments}

The work was sponsored by the Propulsion and Power Division at the NASA-Johnson Space Center. This research used resources of the Advanced Photon Source, a U.S. Department of Energy (DOE) Office of Science User Facility operated for the DOE Office of Science by Argonne National Laboratory under Contract No. DE-AC02-06CH11357. The authors express gratitude to J. Patrick McManamen, Dr. Ben Halls, and Dr. James Gord for technical assistance.

\section{References}

${ }^{1}$ Heindel, T. J., "A review of X-ray flow visualization with applications to multiphase flows," Journal of Fluids Engineering, Vol. 133, No. 7, 2011, pp. 074001.

${ }^{2}$ Linne, M., "Imaging in the optically dense regions of a spray: a review of developing techniques," Progress in Energy and Combustion Science, Vol. 39, No. 5, 2013, pp. 403-440.

${ }^{3}$ Als-Nielsen, J. and McMorrow, D., Elements of modern X-ray physics, John Wiley \& Sons, 2011.

${ }^{4}$ Kastengren, A. and Powell, C. F., "Synchrotron X-ray techniques for fluid dynamics," Experiments in fluids, Vol. 55, No. 3, 2014, pp. 1686.

${ }^{5}$ Halls, B. R., Heindel, T. J., Kastengren, A. L., and Meyer, T. R., "Evaluation of X-ray sources for quantitative two-and three-dimensional imaging of liquid mass distribution in atomizing sprays," International Journal of Multiphase Flow, Vol. 59, 2014, pp. 113-120.

${ }^{6}$ Halls, B., Roy, S., Gord, J., Kastengren, A., and Meyer, T., "Quantitative imaging of single-shot liquid distributions in sprays using broadband flash x-ray radiography," International Journal of Multiphase Flow, Vol. 87, 2016, pp. 241-249.

${ }^{7}$ Mason-Smith, N., Duke, D. J., Kastengren, A. L., Stewart, P. J., Traini, D., Young, P. M., Chen, Y., Lewis, D. A., Soria, J., Edgington-Mitchell, D., et al., "Insights into Spray Development from Metered-Dose Inhalers Through Quantitative X-ray Radiography," Pharmaceutical research, Vol. 33, No. 5, 2016, pp. 1249-1258.

${ }^{8}$ Kastengren, A., Powell, C. F., Dufresne, E. M., and Walko, D. A., "Application of X-ray fluorescence to turbulent mixing," Synchrotron Radiation, Vol. 18, No. 5, 2011, pp. 811-815.

${ }^{9}$ Radke, C. D., Patrick McManamen, J., Kastengren, A. L., Halls, B. R., and Meyer, T. R., "Quantitative time-averaged gas and liquid distributions using x-ray fluorescence and radiography in atomizing sprays," Optics letters, Vol. 40, No. 9, 2015, pp. $2029-2032$. 
${ }^{10}$ Halls, B. R., Meyer, T. R., and Kastengren, A. L., "Quantitative measurement of binary liquid distributions using multiple-tracer x-ray fluorescence and radiography," Optics express, Vol. 23, No. 2, 2015, pp. 1730-1739.

${ }^{11}$ Duke, D. J., Kastengren, A. L., Mason-Smith, N., Chen, Y., Young, P. M., Traini, D., Lewis, D., Edgington-Mitchell, D., and Honnery, D., "Temporally and spatially resolved x-ray fluorescence measurements of in-situ drug concentration in metered-dose inhaler sprays," Pharmaceutical research, Vol. 33, No. 4, 2016, pp. 816-825.

${ }^{12}$ Powel, C., Yue, Y., Gupta, S., McPherson, A., Poola, R., and Wang, J., "Development of a quantitative measurement of a diesel spray core using synchrotron X-rays," Eighth International Conference on Liquid Atomization and Spray Systems, Pasadena, CA, 2000.

${ }^{13}$ MacPhee, A. G., Tate, M. W., Powell, C. F., Yue, Y., Renzi, M. J., Ercan, A., Narayanan, S., Fontes, E., Walther, J., Schaller, J., et al., "X-ray imaging of shock waves generated by high-pressure fuel sprays," Science, Vol. 295, No. 5558, 2002, pp. 1261-1263.

${ }^{14}$ Wang, Y., Im, K.-S., Fezzaa, K., Lee, W., Wang, J., Micheli, P., Laub, C., et al., "Quantitative x-ray phase-contrast imaging of air-assisted water sprays with high Weber numbers," Applied physics letters, Vol. 89, No. 15, 2006, pp. 151913.

${ }^{15}$ Wang, Y., Liu, X., Im, K.-S., Lee, W.-K., Wang, J., Fezzaa, K., Hung, D. L., and Winkelman, J. R., "Ultrafast X-ray study of dense-liquid-jet flow dynamics using structure-tracking velocimetry," Nature Physics, Vol. 4, No. 4, 2008 , pp. 305-309.

${ }^{16}$ Lin, K., Carter, C., Cernucan, J., Fezza, K., and Wang, J., "Ultrafast X-ray study of aerated-liquid jets in a quiescent environment," ILASS Americas, 21st annual conference on liquid atomization and spray systems, Orlando, 2008.

${ }^{17}$ Halls, B. R., Radke, C. D., Reuter, B. J., Kastengren, A. L., Gord, J. R., and Meyer, T. R., "High-speed, two-dimensional synchrotron white-beam x-ray radiography of spray breakup and atomization," Optics Express, Vol. 25, No. 2, 2017, pp. 16051617.

${ }^{18}$ Radke, C. D., Patrick McManamen, J., Kastengren, A. L., Swantek, A. B., and Meyer, T. R., "Synchrotron x-ray interrogation of turbulent gas-liquid mixing in cryogenic rocket sprays (in press)," AIAA, 2017.

${ }^{19}$ del Río, M. S. and Dejus, R. J., "XOP v2. 4: recent developments of the x-ray optics software toolkit," SPIE Optical Engineering+ Applications, International Society for Optics and Photonics, 2011, pp. 814115-814115.

\section{Figures}

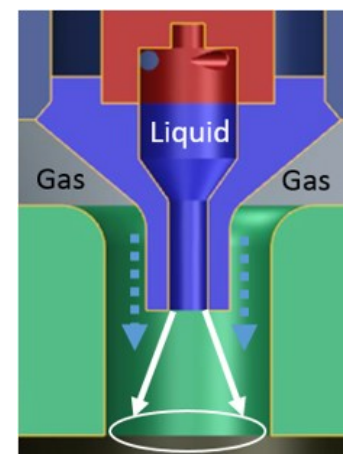

Figure 1. Cross section of the injector used in all experiments.

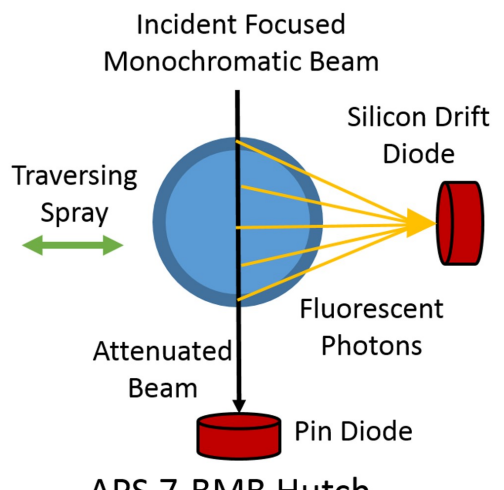

APS 7-BMB Hutch

Figure 2. Experimental setup for simultaneous radiography and fluorescence measurements. 


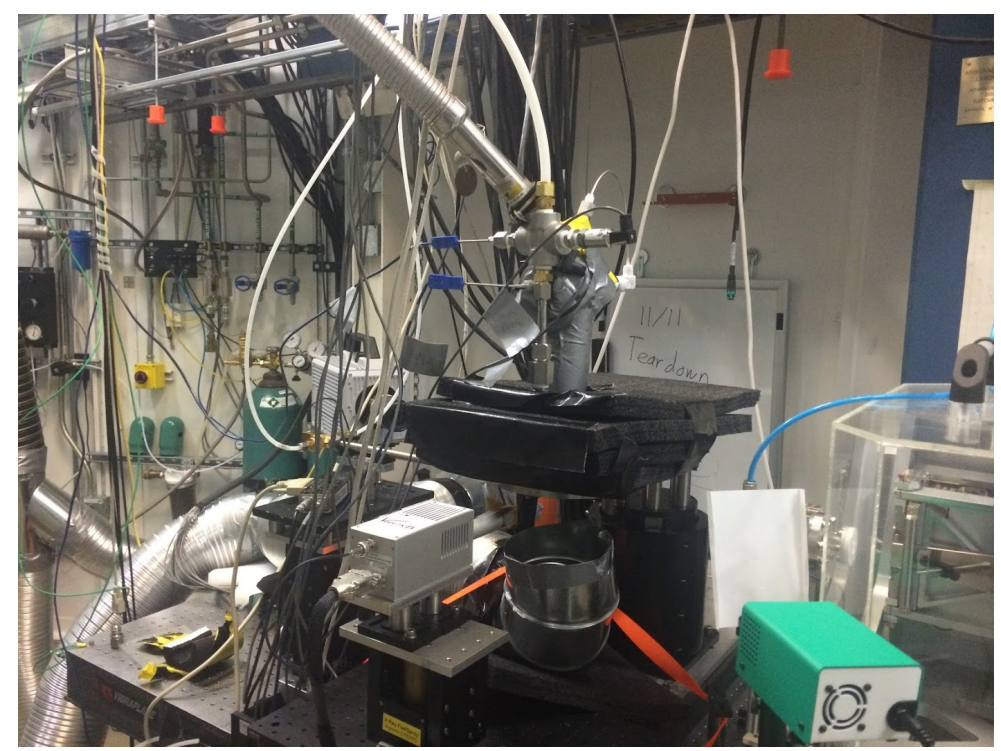

Figure 3. Installed injector showing configuration for cryogenic operations. As can be observed, setup facilitated visible light shadowgraphy, in addition to simultaneous x-ray radiography and fluorescence.

\section{File Scan539.tif, Image 250}

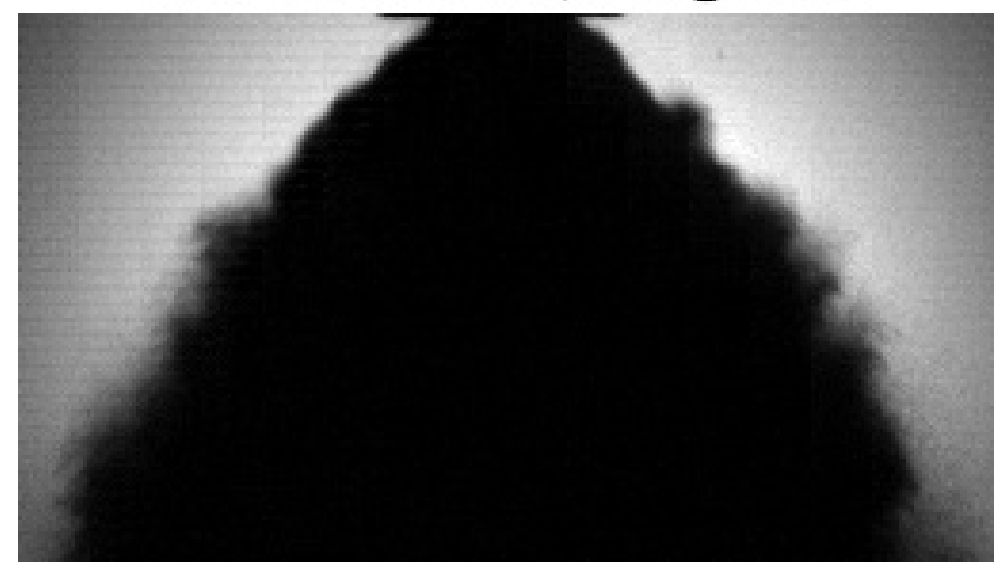

Figure 4. Example shadowgram of flow field created from the pressure swirl atomizer of liquid nitrogen flow collected at $40 \mathrm{kHz}$ with a spatial scale of approximately $30 \mathrm{~mm}$ by $50 \mathrm{~mm}$.

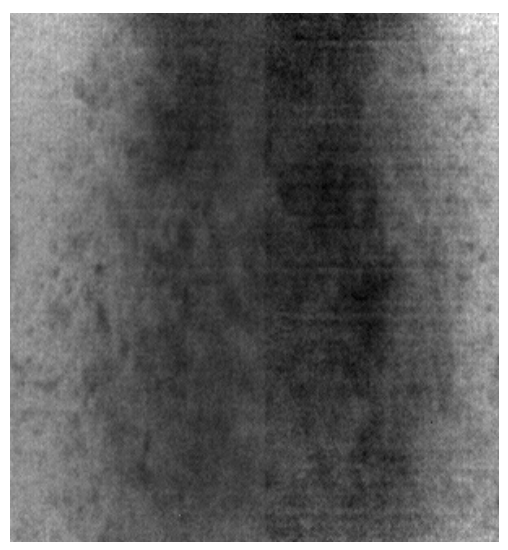

Figure 5. Visible light shadowgraph of liquid water and gas nitrogen taken at $40 \mathrm{kHz}$ with a spatial scale of approximately $30 \mathrm{~mm}$ by $50 \mathrm{~mm}$, are shown for for a gas flow of $R e_{g} \approx 21,500$ 


\section{File Scan583.tif, Image 250}

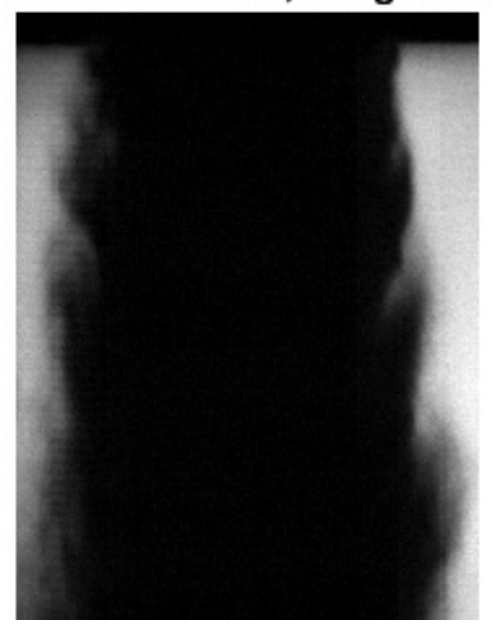

Figure 6. Visible light shadowgraph of liquid nitrogen and gas nitrogen taken at $40 \mathrm{kHz}$ with a spatial scale of approximately $30 \mathrm{~mm}$ by $50 \mathrm{~mm}$, are shown for for a gas flow of $R e_{g} \approx 28,500$.

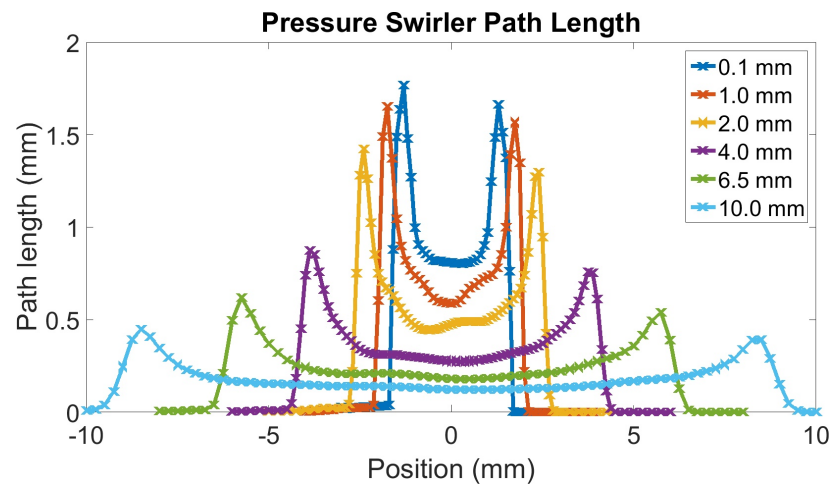

Figure 7. Example plot showing quantitative path length of water spray at different axial locations from the nozzle exit plane at flow of $40.5 \mathrm{~g} / \mathrm{s}$.

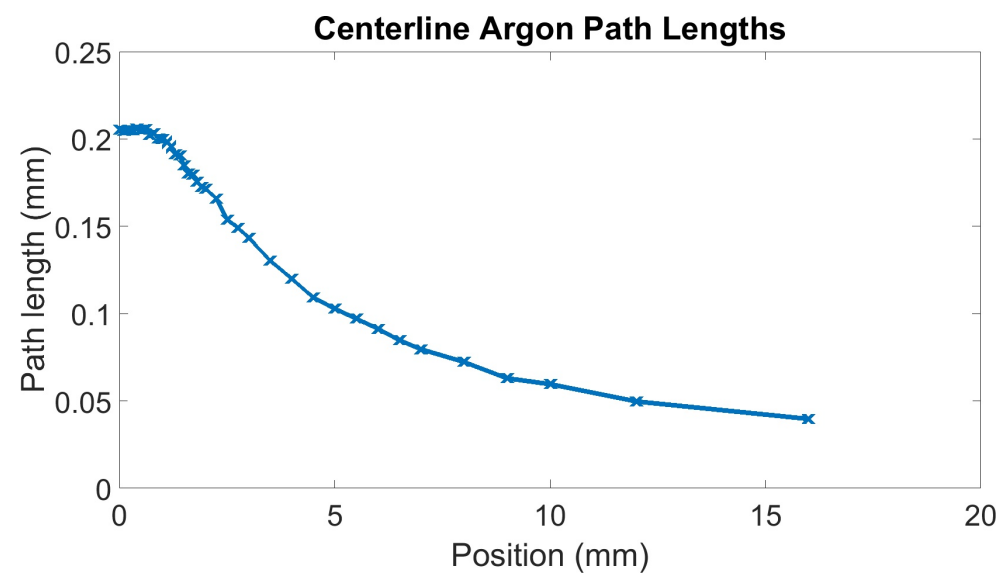

Figure 8. Example plot showing quantitative path length of liquid argon for pressure swirler flows at different axial locations from the nozzle exit plane at flow of $121.2 \mathrm{~g} / \mathrm{s}$. 


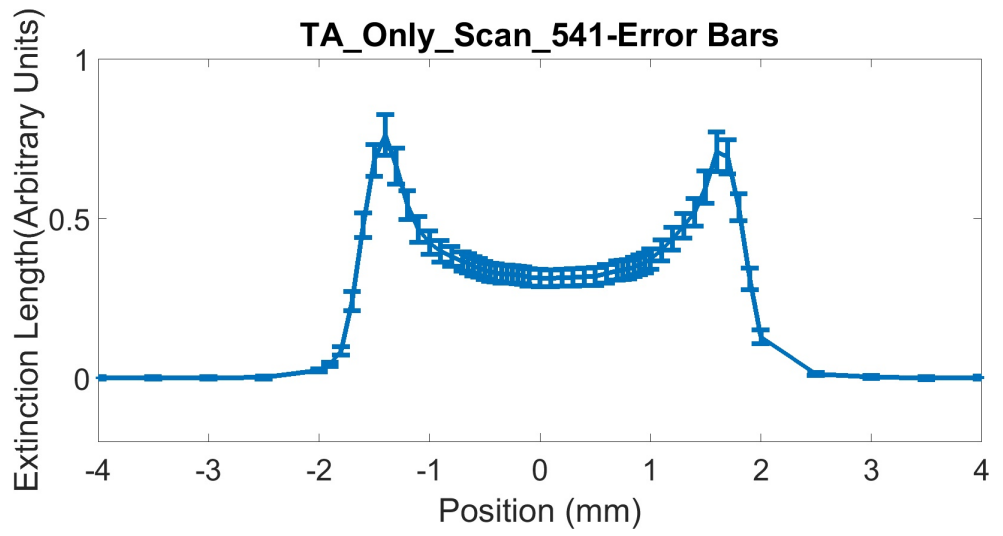

Figure 9. Comparison of uncertainty for liquid nitrogen flow at $\mathbf{X}=\mathbf{0 . 5} \mathbf{~ m m}\left(x / d_{0}=0.16\right)$.

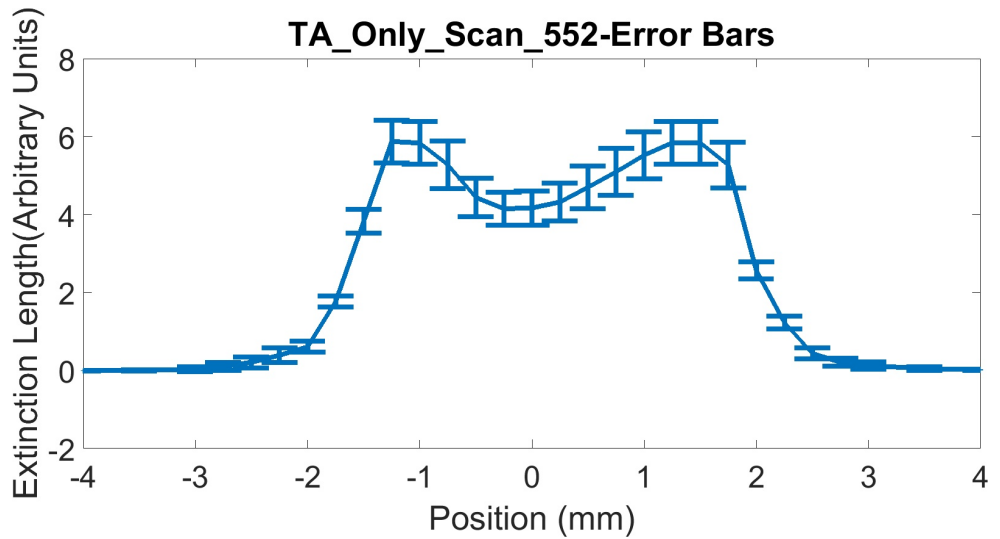

Figure 10. Comparison of uncertainty for liquid argon at $\mathbf{X}=\mathbf{0 . 5} \mathbf{~ m m}\left(x / d_{0}=0.16\right)$.

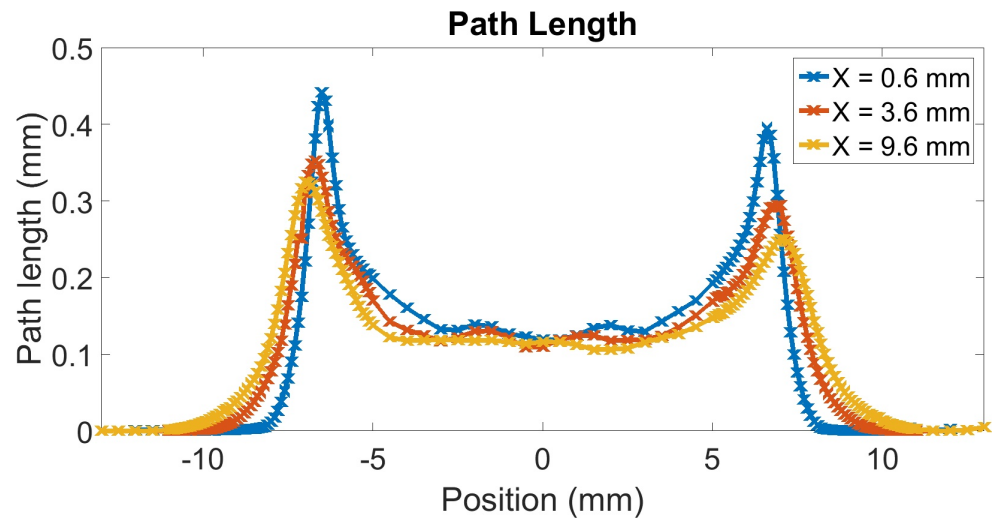

Figure 11. Example plot showing quantitative path length of water spray at an axial location of $\mathrm{X}=0.6 \mathrm{~mm}$ $\left(x / D_{0}=0.06\right), \mathbf{X}=\mathbf{3 . 6} \mathbf{m m}\left(x / D_{0}=0.35\right)$, and $\mathbf{X}=\mathbf{9 . 6} \mathbf{~ m m}\left(x / D_{0}=0.94\right)$ for a water mass flow rate of $41.3 \mathrm{~g} / \mathrm{s}$ and gas flow Reynolds number of $R e_{g} \approx 7,000$. 


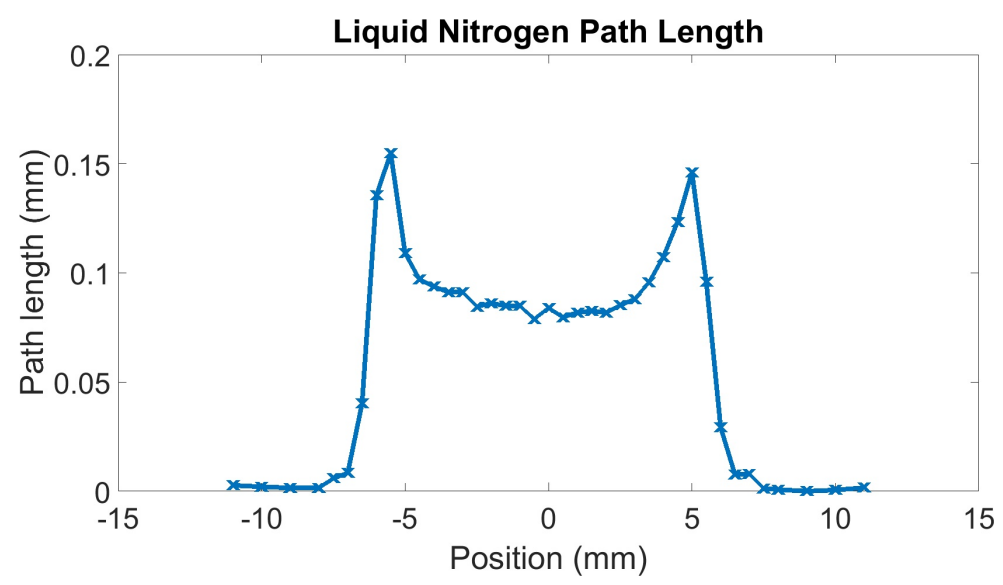

Figure 12. Example plot showing quantitative path length for liquid nitrogen at different gas Reynolds number. Liquid mass flow rate of $\mathbf{8 7 . 7} \mathbf{g} / \mathbf{s}$ and a gas Reynolds number of $R e_{g} \approx 11,000$ at $\mathbf{X}=\mathbf{0 . 5} \mathbf{~ m m}\left(x / D_{0}=0.05\right)$ from the injector exit.
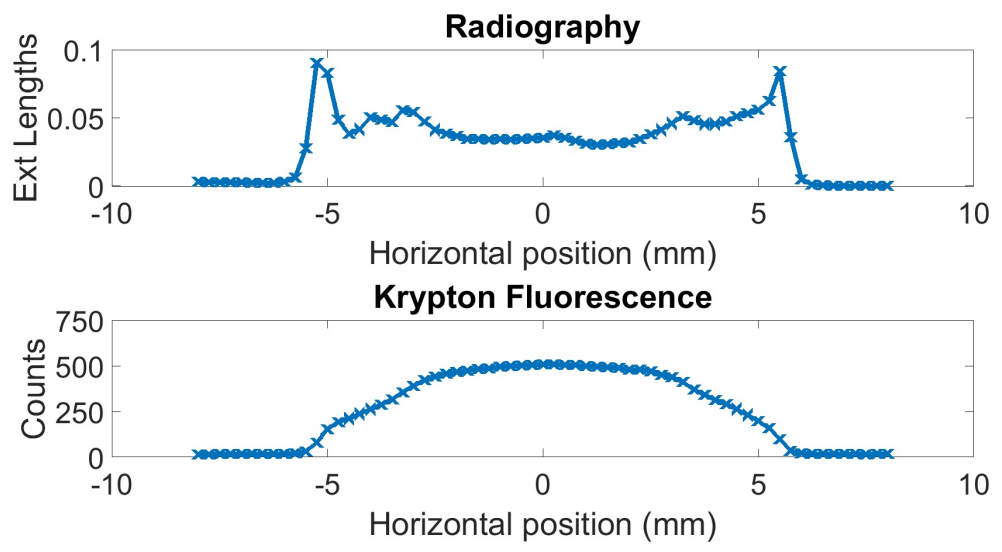

Figure 13. Example line plot from radiographs and krypton fluorescence scan taken at $\mathbf{X}=\mathbf{0 . 5} \mathbf{m m}\left(x / D_{0}=0.05\right)$ from the injector exit

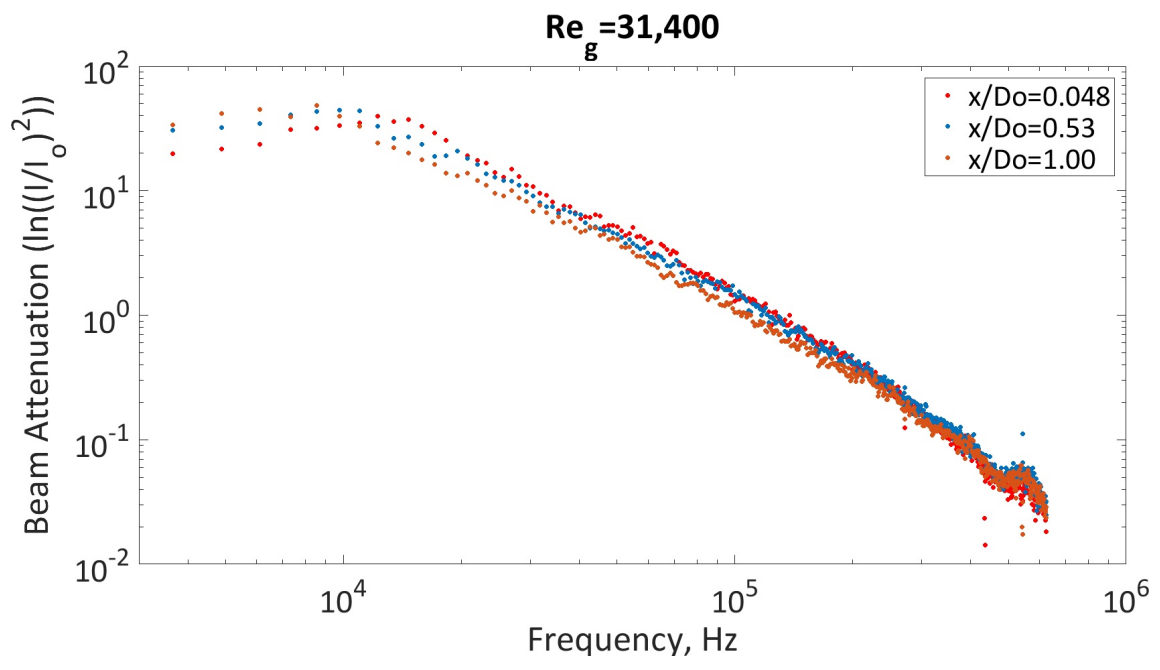

Figure 14. Example plot of spectra from time-resolved spectra for liquid nitrogen and gaseous argon. Plots of centerline spectra shown for $x / D_{O}=0.048, x / D_{O}=0.53, x / D_{O}=1.00$. 


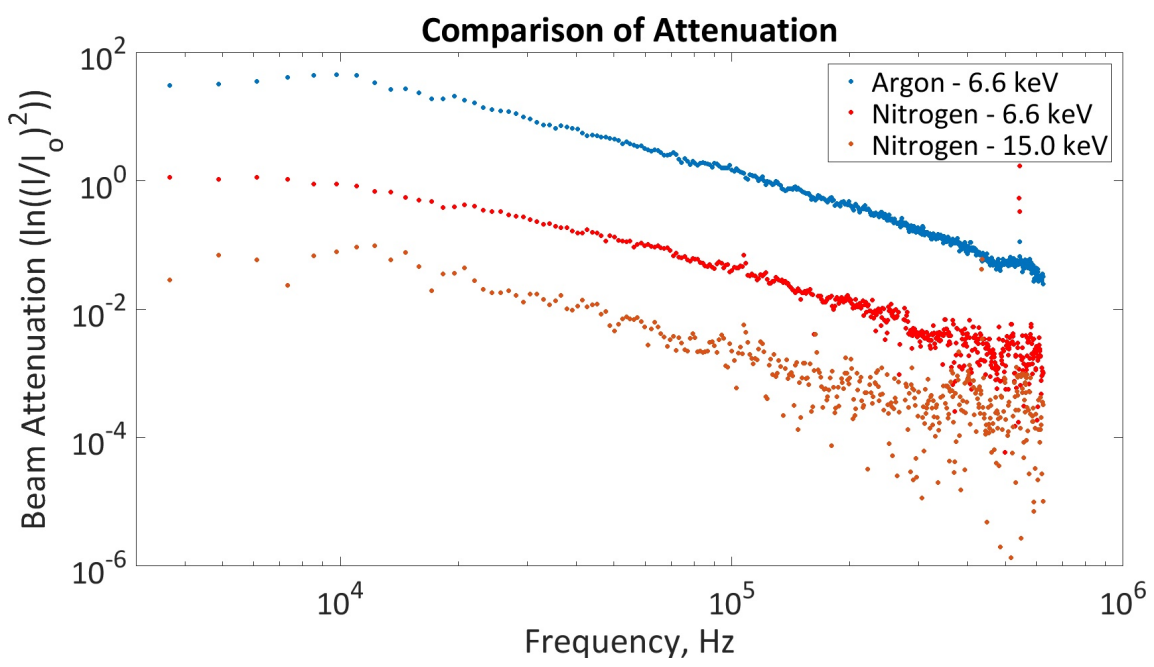

Figure 15. Plot comparing centerline time-resolved scans of different media and at different beam energies, but for comparable flow conditions. All scans collected at $\mathbf{X}=\mathbf{5 . 6} \mathbf{~ m m}\left(x / D_{O}=0.53\right)$. Plot shows comparison of attenuation for argon at $6.6 \mathrm{keV}$, nitrogen at $6.6 \mathrm{keV}$ and nitrogen at $15.0 \mathrm{keV}$.

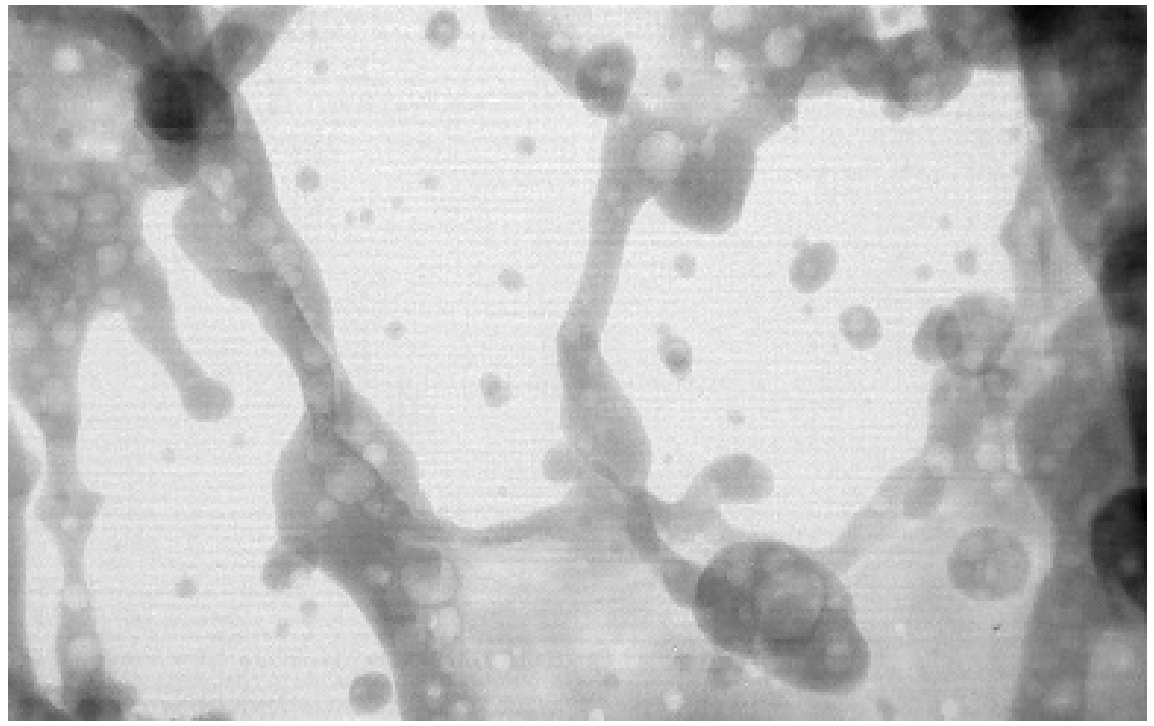

Figure 16. Example polychromatic radiograph taken at $120 \mathrm{kHz}$ in an optically dense region of the spray showing many complex internal features such as hollow droplets and complex internal ligaments. The spatial field of view of the radiographs is approximately $8 \mathrm{~mm} \times 6 \mathrm{~mm}$. 